J. Indones. Math. Soc. Vol. 25, No. 02 (2019), pp. 121-127.

\title{
The Probability That an Ordered Pair of Elements is an Engel Pair
}

\author{
S. M. JAFARIAN Amiri ${ }^{1}$, H. Rostami ${ }^{2}$ \\ ${ }^{1}$ Department of Mathematics, University of Zanjan, P.O.Box 45371-38791, \\ Zanjan, Iran, sm_jafarian@znu.ac.ir \\ ${ }^{2}$ Department of Mathematics, University of Zanjan, P.O.Box 45371-38791, \\ Zanjan, Iran, h.rostami5991@gmail.com
}

\begin{abstract}
Let $G$ be a finite group. We denote by $e p(G)$ the probability that $\left[x,_{n} y\right]=1$ for two randomly chosen elements $x$ and $y$ of $G$ and some positive integer $n$. For $x \in G$ we denote by $E_{G}(x)$ the subset $\{y \in G:[y, n x]=$ 1 for some integer $n\}$. $G$ is called an $E$-group if $E_{G}(x)$ is a subgroup of $G$ for all $x \in G$. Among other results, we prove that if $G$ is an non-abelian $E$-group with $e p(G)>\frac{1}{6}$, then $G$ is not simple and minimal non-solvable.

Key words : finite group, E-group, Engel element.
\end{abstract}

Abstrak. Misalkan $G$ meruapakan suatu grup hingga. Misalkan juga ep $(G)$ merupakan peluang dari $[x, n y]=1$ untuk dua unsur yang dipilih secara random $x$ dan $y$ di $G$ dan suatu bilangan bulat positif $n$. Untuk $x \in G$, misalkan $E_{G}(x)$ merupakan suatu subset $\left\{y \in G:\left[y,{ }_{n} x\right]=1\right.$ for some integer $\left.n\right\}$. Grup $G$ disebut suatu $E$-group jika $E_{G}(x)$ merupakan suatu subgrup dari $G$ untuk semua $x \in G$. Salah satu hasil dalam artikel ini adalah, dibuktikan bahwa jika $G$ merupakan suatu nonabelian $E$-group dengan $e p(G)>\frac{1}{6}$, maka $G$ bukan merupakan suatu grup simpel dan minimal non-solvable.

Kata kunci : grup hingga, E-group, unsur Engel.

\section{INTRODUCTION}

Let $G$ be any group and $x_{1}, \cdots, x_{n} \in G$. We define $\left[x_{1}, x_{2}\right]=x_{1}^{-1} x_{2}^{-1} x_{1} x_{2}$ and for $n \geq 2$ inductively $\left[x_{1}, \cdots, x_{n}\right]$ as follows:

$$
\left[x_{1}, \cdots, x_{n}\right]=\left[x_{1}, \cdots, x_{n-1}\right]^{-1} x_{n}^{-1}\left[x_{1}, \cdots, x_{n-1}\right] x_{n} .
$$

2000 Mathematics Subject Classification: Primary: 20F05; Secondary: 05C05. Received: 08-10-2017, revised: 15-10-2018, accepted: 21-10-2018. 
If $x_{2}=\cdots=x_{n}$, then we denote $\left[x_{1}, \cdots, x_{n}\right]$ by $\left[x_{1, n-1} x_{2}\right]$. If there are two elements $x$ and $y$ in $G$ such that $\left[y,_{n} x\right]=1$ for some integer $n>0$, then $x$ is called a left Engel element with respect to $y$. An element $x$ of $G$ is called a left Engel element if for all $y \in G$ there exists an integer $n=n(x, y)>0$ such that $\left[y_{n} x\right]=1$. The engelizer of $x$ in $G$ is defined to be the subset $E_{G}(x)=\{y \in$ $G:\left[y{ }_{n} x\right]=1$ for some $\left.n\right\}$. We notice that $E_{G}(x)$ is not necessary a subgroup of $G$. A group $G$ is called an $E$-group if $E_{G}(x)$ is a subgroup of $G$ for all $x \in G$. In particular, $G$ is Engel if $E_{G}(x)=G$ for all $x \in G$. In [15] Peng introduced and investigated the structure of finite solvable $E$-groups and also he generalized the previous results in [14]. After him other group theorists such as Heineken and Casolo studied E-groups (see [5], [6] and [12]). They determined simple $E$-groups. In this paper we focus on $E$-groups with a different viewpoint. For this reason, we conider some generalization of the commutativity degree of a finite group $G$, which means the probability that two randomly chosen elements of $G$ commute. In other words

$$
c p(G)=\frac{|\{(x, y) \in G \times G:[x, y]=1\}|}{|G|^{2}} .
$$

In the literature, the commutativity degree of a group $G$ denoted variously by $d(G)$, $\operatorname{pr}(G)$ or $c p(G)$ and are studied and generalized by some authors (see [11], [8], [10]). Note that $c p(G)=\frac{\sum_{x \in G}\left|C_{G}(x)\right|}{|G|^{2}}$ where $C_{G}(x)=\{y \in G:[y, x]=1\}$ and we know that $C_{G}(x)$ is a subgroup of $G$ for every $x \in G$.

Since $c p\left(A_{5}\right)=\frac{1}{12}$, J. Dixon observed that $c p(G) \leq \frac{1}{12}$ for any finite nonabelian simple group $G$ (see [9]) and Guralnick and Robinson [9] extended and proved that every group $G$ with $c p(G)>\frac{1}{12}$ is solvable. Now by considering $c p(G)$ and what are said in above, for all finite groups $G$, we define $\operatorname{ep}(G)$ to be the probability of being left Engel element in a group $G$,i.e.,

$$
e p(G)=\frac{\mid\{(x, y) \in G \times G ;[x, n y]=1 \text { for some natural number } n\} \mid}{|G|^{2}} .
$$

Also it is evident that $\operatorname{ep}(G)=\frac{\sum_{x \in G}\left|E_{G}(x)\right|}{|G|^{2}}$. But $E_{G}(x)$ is not necessary a subgroup of $G$ and so it is difficult to obtain information about a group $G$ from $e p(G)$. Next since $\operatorname{ep}\left(A_{5}\right)=\frac{1}{6}$ (see Proposition 2.6), it is natural that we propose the following question:

Question 1.1. If $G$ is a finite group with ep $(G)>\frac{1}{6}$, then is $G$ solvable?

In the present paper, we will show that if $G$ is an $E$-group, then it is not simple and minimal non-solvable.

Theorem 1.2. Let $G$ be a non-abelian E-group with ep $(G)>\frac{1}{6}$. Then $G$ is not simple and minimal non-solvable.

In this article all groups are finite and an engelizer in a group means the engelizer of some element of the group. We denote by $\operatorname{PSL}(2, q)$ and $S z(q)$ the projective special linear group of degree two over the finite field of size $q$ and the Suzuki group over the finite field of size $q=2^{2 m+1}$ with $m>0$, respectively. Also 
$C_{n}, C_{2}^{n}$ and $D_{2 n}$ denote the cyclic group of order $n$, the elementary abelian 2group of rank $n$ and the dihedral group of order $2 n$, respectively. Other notation is standard and can be found in [16].

\section{Main Results}

The following result has been proved by Heineken and Casolo in [12] and [5]. This will be used in proof of Theorem 1.2.

Theorem 2.1. A non-abelian simple group is an E-group if and only if it is one of the following groups:

$P S L\left(2,2^{n}\right), \quad n \geq 2 ; \quad S z\left(2^{2 m+1}\right)$ with $m>0$.

Recall that a collection $S$ of proper subgroups of a group $G$ is called a partition if every nonidentity element of $G$ belongs to a unique subgroup in $S$. In what follows we determine the structure of all engelizers in a Suzuki group.

Lemma 2.2. Let $G=S z(q)$ and $r=\sqrt{\frac{q}{2}}$. If $1 \neq x \in G$, then $E_{G}(x)$ has one of the following structures.

1. The Frobenius group $P \rtimes C_{q-1}$ where $P$ is Sylow 2-subgroup of $G$;

2. The dihedral group $D_{2(q-1)}$;

3. The Frobenius group $C_{q-2 r+1} \rtimes C_{4}$;

4. The Frobenius group $C_{q+2 r+1} \rtimes C_{4}$.

Proof. By Theorem 2.1, $G$ is an $E$-group and so $E_{G}(x)$ is a subgroup of $G$. If $E_{G}(x)=G$, then $x$ is a left Engel element of $G$ and therefore since $G$ is finite, by Corollary 3.17 of [3], $x$ belongs to the Hirsch-Plotkin radical of $G$, a contradiction. Therefore $E_{G}(x)$ is a proper subgroup of $G$. By Theorems 3.10 and 3.11 of [13], $\Gamma=\left\{P^{g}, A^{g}, B^{g}, C^{g}: g \in G\right\}$ is a partition of $G$ where $P$ is a Sylow 2-subgroup $G$, $A$ is cyclic of order $q-1, B$ is cyclic of order $q-2 r+1$ and $C$ is cyclic of order $q+2 r+1$. It follows that $x \in M$ for some $M \in \Gamma$. Since $A, B$ and $C$ are cyclic and $P$ is nilpotent, then for all $y \in M$ we have $M=E_{M}(y)$, hence $M \leq E_{G}(x)$. Let $K$ be a maximal subgroup of $G$ containing $E_{G}(x)$. Then $K$ is isomorphic to $P \rtimes C_{q-1}, C_{q-2 r+1} \rtimes C_{4}, C_{q+2 r+1} \rtimes C_{4}, D_{2(q-1)}$ or $S z\left(q_{0}\right)$ where $q_{0}^{l}=q, l$ is prime and $q_{0}>2$ (see for example page 343 of [4]). Now we consider the following three cases.

Case 1. If $M$ is a Sylow 2-subgroup of $G$, then $K=M \rtimes A^{g}$ for some $g \in G$ and also $M^{\prime}=Z(M)$ by Claim 3.2 of [4]. Since $\frac{K}{M}$ is a cyclic group, $K^{\prime} \leq M$. Now if $y \in K$, then $\left[y,,_{2} x\right] \in M^{\prime}$ which implies $\left[y,_{3} x\right]=1$. Consequently $E_{G}(x)=K$, as a desired.

Case 2. Suppose that $M$ is cyclic of order $q-1$. By page 137 of [18], we see that $K=M \rtimes\langle a\rangle \cong D_{2(q-1)}$ for some involution $a \in G$. It follows that $[a, x] \in M$ and since $M$ is cyclic, we have $\left[a,{ }_{2} x\right]=1$ which yields that $a \in E_{G}(x)$. Consequently $E_{G}(x)=K$, as wanted.

Case 3. Suppose that $M$ is a cyclic group of order either $q-2 r+1$ or $q+2 r+1$. Then $K=M \rtimes H$ where $H$ is a cyclic group of order 4 . Similarly if $a \in H$, then $\left[a,{ }_{2} x\right]=1$ and so $E_{G}(x)=K$. This completes the proof. 
In the following lemma, we compute $e p(S z(q))$.

Proposition 2.3. $\operatorname{ep}(S z(q))=\frac{q^{2}+3 q-2}{q^{2}\left(q^{2}+1\right)(q-1)}$ where $q=2^{2 n+1}, n>0$. In particular $\operatorname{ep}(S z(q)) \leq \frac{1}{6}$.

Proof. Let $G=S z(q)$. For a given subgroup $H$ of $G$, we denote the set of all conjugates of $H$ in $G$ by $C l(H)$. Let $\Gamma$ be the partition of $\mathrm{G}$ described in the proof of Lemma 2.2. If $1 \neq x \in G$, then $x \in M$ for some $M \in \Gamma$. If $M=P^{g}$ for some $g \in G$, then by using Lemma 2.2 and simple calculation one can see that $\left|E_{G}(x)\right|=q^{2}(q-1)$. But the number of Sylow 2-subgroups of $G$ is $q^{2}+1$ ( see Theorems 3.10 and 3.11 of chapter XI in [13] or proof of Theorem 1.2 of [2]). It follows that

$$
\sum_{X \in C l(P)} \sum_{1 \neq x \in X}\left|E_{G}(x)\right|=q^{2}(q-1)\left(q^{2}+1\right)\left(q^{2}-1\right) .
$$

If $M=A^{g}$ for some $g \in G$, then $\left|E_{G}(x)\right|=2(q-1)$. Since the number of conjugates of $A$ in $G$ is $\frac{q^{2}\left(q^{2}+1\right)}{2}$ ( see Theorems 3.10 and 3.11 of chapter XI in [13] or proof of Theorem 1.2 of [2]), we have

$$
\sum_{X \in C l(A)} \sum_{1 \neq x \in X}\left|E_{G}(x)\right|=\frac{q^{2}\left(q^{2}+1\right)}{2}(q-2) 2(q-1) .
$$

If $M=B^{g}$ for some $g \in G$, then $\left|E_{G}(x)\right|=4(q-2 r+1)$. But the number of conjugates of $B$ in $G$ is $\frac{q^{2}(q-1)\left(q^{2}+1\right)}{4(q-2 r+1)}$ ( see Theorems 3.10 and 3.11 of chapter XI in [13] or proof of Theorem 1.2 of [2]). It follows that

$$
\sum_{X \in C l(B)} \sum_{1 \neq x \in X}\left|E_{G}(x)\right|=q^{2}(q-1)\left(q^{2}+1\right)(q-2 r) .
$$

If $M=C^{g}$ for some $g \in G$, then $\left|E_{G}(x)\right|=4(q+2 r+1)$. But the number of conjugates of $C$ in $G$ is $\frac{q^{2}(q-1)\left(q^{2}+1\right)}{4(q+2 r+1)}$ ( see Theorems 3.10 and 3.11 of chapter XI in [13] or proof of Theorem 1.2 of [2]). So

$$
\sum_{X \in C l(C)} \sum_{1 \neq x \in X}\left|E_{G}(x)\right|=q^{2}(q-1)\left(q^{2}+1\right)(q+2 r) .
$$

Consequently

$$
e p(G)=\frac{\sum_{x \in G}\left|E_{G}(x)\right|}{|G|^{2}}=\frac{|G|+\sum_{1 \neq x \in G}\left|E_{G}(x)\right|}{\left(q^{2}\left(q^{2}+1\right)(q-1)\right)^{2}}=\frac{q^{2}+3 q-2}{q^{2}\left(q^{2}+1\right)(q-1)} .
$$

According to a well known theorem of [7]( see chapter 12 pages 260-287), the maximal subgroups of $\operatorname{PSL}\left(2,2^{n}\right),(n>1)$ fall into four families as follows.

Theorem 2.4. Every maximal subgroup of $G=P S L(2, q)$ with $q=2^{n}>3$ is isomorphic to one of the following.

1. $C_{2}^{n} \rtimes C_{q-1}$, that is, the stabilizer of a point of the projective line; 
2. Dihedral group $D_{2(q-1)}$;

3. Dihedral group $D_{2(q+1)}$;

4. Projective general linear group $P G L\left(2, q_{0}\right)$ for $q=q_{0}^{r}$ with $r$ a prime and $q_{0} \neq 2$.

Lemma 2.5. Let $G=P S L\left(2,2^{n}\right)$ such that $n>1$ and $1 \neq x \in G$. Then $E_{G}(x)$ is isomorphic to $D_{2(q-1)}, D_{2(q+1)}$ or $C_{2}^{n} \rtimes C_{q-1}$.

Proof. It follows from Theorem 2.1 that $E_{G}(x)$ is a subgroup of $G$. By Proposition 3.21 of [1], $G$ has a partition $\Gamma=\left\{P^{g}, A^{g}, B^{g} \mid g \in G\right\}$ such that $P$ is an elementary abelian 2- group of order $q, A$ is cyclic of order $q-1$ and $B$ is cyclic of order $q+1$. Therefore $x \in M$ where $M \in \Gamma$ and since $P, A$ and $B$ are abelian groups, we have $M \leq E_{G}(x)$ ( Note that since $G$ is finite, $E_{G}(x) \neq G$ by exercise 12.3.2 of [16]( see also Theorem 3.14 and Corollary 3.17 of [3]).

Case 1. Let $M=A^{g}$ for some $g \in G$. By Proposition 3.21 of [1], we have normalizer of $\langle x\rangle$ in $G ; N_{G}(x)$ is a dihedral group of order $2(q-1)$ and so by Theorem 2.4, it is a maximal subgroup of $G$. Therefore $N_{G}(x)=M \rtimes\langle a\rangle$ for some involution $a \in G$. It follows that $a \in E_{G}(x)$ and hence $E_{G}(x)=N_{G}(x) \cong D_{2(q-1)}$, as wanted.

Case 2. Let $M=B^{g}$ for some $g \in G$. Similarly to case 1 , we get $E_{G}(x) \cong$ $D_{2(q+1)}$.

Case 3. Let $M=P^{g}$ for some $g \in G$. Then $M$ is contained in a maximal subgroup $T$ such that $T=M \rtimes L$ and $L$ is cyclic of order $q-1$ by Theorem 2.4. Since $x \in M$, we have $E_{G}(x)=T$. This completes the proof.

In the following we compute $\operatorname{ep}\left(\operatorname{PSL}\left(2,2^{n}\right)\right)$ for $n>1$.

Proposition 2.6. If $q>3$ is even, then $\operatorname{ep}(P S L(2, q))=\frac{3 q-2}{q(q-1)(q+1)}$. In particular $e p\left(P S L\left(2,2^{n}\right)\right) \leq e p\left(A_{5}\right)=\frac{1}{6}$.

Proof. Let $G=P S L(2, q)$. Then $G$ has a partition

$$
\Gamma=\left\{P^{g}, A^{g}, B^{g} \mid g \in G\right\}
$$

which is the same as in the proof of Lemma 2.5. Also by Lemma 2.5, $E_{G}(x)$ is a maximal subgroup of $G$ for any $1 \neq x \in G$. But by Proposition 2.4 of [2], the numbers of conjugates of $A, B$ and $P$ in $G$ are $\frac{q(q+1)}{2}, \frac{q(q-1)}{2}$ and $q+1$, respectively. Therefore we have

$$
\begin{gathered}
\sum_{X \in C l(A)} \sum_{1 \neq x \in X}\left|E_{G}(x)\right|=2(q-1)(q-2) \frac{q(q+1)}{2}=(q-1) q(q+1)(q-2), \\
\sum_{X \in C l(B)} \sum_{1 \neq x \in X}\left|E_{G}(x)\right|=2(q+1) q \frac{q(q-1)}{2}=(q-1) q^{2}(q+1)
\end{gathered}
$$

and also

$$
\sum_{X \in C l(P)} \sum_{1 \neq x \in X}\left|E_{G}(x)\right|=q(q-1)(q-1)(q+1)=(q-1)^{2} q(q+1) .
$$


It follows that $\operatorname{ep}(G)=\frac{\sum_{x \in G}\left|E_{G}(x)\right|}{|G|^{2}}=\frac{|G|+\sum_{1 \neq x \in G}\left|E_{G}(x)\right|}{((q-1) q(q+1))^{2}}=\frac{3 q-2}{q(q-1)(q+1)}$, as desired.

The following lemma due to Heineken, will be used in the proof of the main result.

Lemma 2.7. Let $G$ be an E-group and $N \unlhd G$. Then both of $N$ and $G / N$ are E-groups.

Proof. Theorem 1 of [12] and its proof give the result.

Lemma 2.8. Let $G$ be a group and $N \unlhd G$. Then ep $(G) \leq e p(G / N)$.

Proof. Let $S=\{(a, b) \in G \times G ;(a, b)$ is an Engel pair $\}$ and $\bar{S}=\{(A, B) \in G / N \times$ $G / N ;(A, B)$ is an Engel pair $\}$. Now the subsets $A \times B$ for $(A, B) \in \bar{S}$ are pairwise disjoint subsets of $G \times G$ where each has size $|N| .|N|$. Clearly $S \subseteq \bigcup_{(A, B) \in \bar{S}} A \times B$ and thus $|S| \leq|\bar{S}| \cdot|N|^{2}$ from which it follows that

$$
e p(G)=\frac{|S|}{|G|^{2}} \leq \frac{|\bar{S}| \cdot|N|^{2}}{|G|^{2}}=e p(G / N)
$$

\section{Proof of Theorem 1.2.}

Let $G$ be a non-abelian simple $E$-group. Then by Theorem $2.1, G \cong P S L\left(2,2^{n}\right), n \geq$ 2 or $G \cong S z\left(2^{2 m+1}\right)$ with $m>0$. Now assuming $\operatorname{ep}(G)>\frac{1}{6}$ is on the contrary to Lemmas 2.3 and 2.6 .

Now let $G$ be a minimal non-solvable $E$ - group with $e p(G)>\frac{1}{6}$. By Corollary 1 of [17] we know that if $G$ is a minimal non-solvable group, then for some normal subgroup $N$ of $G, G / N$ is isomorphic to one of the following groups:

1. $\operatorname{PSL}\left(2,2^{p}\right), p$ a prime.

2. $P S L 2\left(3^{p}\right), p$ an odd prime.

3. $\operatorname{PSL}(2, p), p>3$ a prime congruent to 2 or $3 \bmod 5$.

4. $S z\left(2^{p}\right), p$ an odd prime.

5. $\operatorname{PSL}(3,3)$.

Also Lemma 2.7 shows that if $G$ is an $E$-group $G / N$ is an $E$-group too where $N$ is a normal subgroup of $G$. Next since by Theorem 2.1 , only simple groups which are $E$ - groups are $P S L\left(2,2^{n}\right), \quad n \geq 2 ; \quad S z\left(2^{2 m+1}\right)$ with $m>0, G / N$ can be only isomorphic to one of the cases 1 or 4 in above. But for these cases, from Lemmas 2.3 and 2.6 we have $e p(G / N) \leq \frac{1}{6}$. Next, since by Lemma 2.8, $e p(G) \leq e p(G / N)$, we have also that $\operatorname{ep}(G) \leq \frac{1}{6}$. Therefore we get a contradiction by our assumption and proof is complete. 


\section{REFERENCES}

[1] A. Abdollahi, S. Akbari and H. R. Maimani, Non-commuting graph of a group, J. Algbera, 298 (2006), 468-492.

[2] A. Abdollahi, A. Azad, A. Mohammadi HasanAbadi and M. Zarrin, On the clique numbers of non-commuting graphs of certain groups, Algebra Colloquium, 17:4(2010), 611-620 .

[3] A. Abdollahi, Engel elements in groups, Groups St Andrews 2009 in Bath, Volume 1( 2011), 94-117.

[4] S. Aivazidis, On the subgroup permutability degree of the simple Suzuki groups, Monatsh. Math. 176 (2015), 335-358.

[5] C. Casolo, Finite groups in which subnormalizers are subgroups, Rend. Semin. Mat. Univ. Padova 82 (1989), 25-53.

[6] C. Casolo, Subnormalizers in finite groups, Comm. Algebra, 18 (11) (1990), 3791-3818.

[7] L. E. Dickson, Linear Groups: With an Exposition of the Galois Field Theory, Dover Publishing, Inc. New York, 1958.

[8] A. Erfanian, and M. Farrokhi Dg, On the probability of being a 2-Engel group, International Journal of Group Theory 2.4 (2013), 31-38.

[9] R. M. Guralnick and G. R. Robinson, On the commuting probability in finite groups, Journal of Algebra, 300 (2006), 509-528.

[10] E. Khamseh, M.R.R. Moghaddam and F.G. Russo, Some Restrictions on the Probability of Generating Nilpotent Subgroups, Southeast Asian Bulletin of Mathematics, 37.4 (2013)

[11] R. Heffernan, D. MacHale and N SH, Restrictions on commutativity ratios in finite groups, International Journal of Group Theory 3.4 (2014), 1-12.

[12] H. Heineken, On E-groups in the sense of Peng, Glasg. Math. J., 31 (1989), 231-242.

[13] B. Huppert and N. Blackburn, Finite groups, III, Springer-Verlag, Berlin, 1982.

[14] T. A. Peng, On groups with nilpotent derived groups, Arch. Math. 20 (1969), 251-253.

[15] T. A. Peng, Finite soluble groups with an Engel condition, J. Algebra 11 (1969), 319-330.

[16] D. J. S. Robinson, A course in the theory of groups, Springer-Verlag New York 1996.

[17] J. G. Thompson, Nonsolvable finite groups all of whose local subgroups are solvable, Bulletin of the American Mathematical Society 74.3 (1968): 383-437.

[18] M. Suzuki, On a class of doubly transitive groups, Annals of Mathematics (1962), 105-145. 